Molecular

Syndromology
Editorial - Late Breaking Chromosomes

Mol Syndromol 2022;13:85-87

DOI: 10.1159/000522096

\title{
Deep White Matter Cysts in a Patient with Aicardi-Goutières Syndrome and SAMHD1 Variants
}

Children appearing healthy at birth but developing disease symptoms within the first weeks or months of life are among the most challenging in the clinic. The deterioration of their condition calls for rapid diagnosis and swift action, while on the other hand a slowly unfolding, and probably incomplete clinical picture, renders any diagnosis difficult. Often an infection in utero is suspected, and tests for toxoplasmosis, rubella, cytomegalovirus, herpes simplex virus, among others, are initiated. In a recent review, the diagnostic yield of these tests turned out to be limited, however [Fitzpatrick et al., 2021]. Alternatively, a neurologic disorder, such as Landau-Kleffner or Aicardi-Goutières syndrome (AGS), is presumed. This paradigm prompts extensive neurologic investigations and cranial computer tomography (CT) scans or magnetic resonance imaging (MRI) of the brain.

Trying to diagnose a boy born in good condition, but soon showing poor growth and weight gain and developing unprovoked irritability, Oleksy et al. pursued both hypotheses [Oleksy et al., 2022]. First, a screen for the most common congenital viral infections proved negative. Then, metabolic screens of urinary organic acids, serum amino acids and very-long-chain fatty acids, lysosomal enzyme activities, tests for congenital disorders of glyco-

karger@karger.com

(c) 2022 S. Karger AG, Basel

www.karger.com/msy

Karger! sylation and array comparative genomic hybridization showed no abnormalities. Transfontanelle ultrasonography suggested calcifications in the right frontal lobe. CT and brain MRI revealed increased subcortical signal intensities mostly in the frontal and temporal lobes. Concomitantly, the patient showed axial hypotonia and hypertonia of his limbs. Neurological examination at 4 years of age revealed impaired psychomotor development. Overall, the patient was profoundly disabled with a mixed pyramidal and extrapyramidal syndrome and acquired microcephaly. While these findings were consistent with AGS, the absence of chilblains, skin or mucosal ulcers, and glaucoma cast doubt on this clinical diagnosis. Therefore, based on the patient's history, clinical picture, MRI and CT scans, the authors suspected nonprogressive leukoencephalopathy with temporal cysts. Searching for a possible genetic cause, the authors performed whole exome sequencing, which uncovered a p.(Gln235*)/ c. $703 \mathrm{C}>\mathrm{T}$ ) variant in the sterile alpha motif $\mathrm{HD}$ domain 1 (SAMHD1) gene. This variant was inherited from the mother, while the father was a heterozygous carrier of a p.(Phe165Ser) variant. Thus, combining the clinical characteristics of the patient and the SAMHD1 variants, a diagnosis of AGS5 (MIM \# 612952) was rendered. 
Jean Aicardi and Françoise Goutières described 8 children with an early onset, progressive, and severe neurologic disorder and imaging features of intracranial calcification, leukoencephalopathy, cerebral atrophy, and a cerebrospinal fluid pleocytosis [Aicardi and Goutières, 1984]. In 1988, elevated levels of cerebrospinal fluid and serum interferon- $\alpha$ were demonstrated in these patients [Lebon et al., 1988]. In further subsequent cases, cutaneous features and autoimmune disease were observed. Thereafter, the disorder became known as AGS. Meanwhile, pathogenic variants in either one of the TREX1 (AGS1), RNASEH2A (AGS2), RNASEH2B (AGS3), RNASEH2C (AGS4), SAMHD1 (AGS5), ADAR1 (AGS6), and IFIH1 (AGS7), and LSM11, and RNU7-1 genes were found in AGS patients [Crow et al., 2006a, b; Rice et al., 2009, 2012, 2014; Livingston and Crow, 2016]. These genes encode proteins involved in degradation of certain types of nucleic acids, such as dsRNAs. In most AGS patients, pathogenic variants exhibit autosomal recessive inheritance, albeit that a few dominant alleles of TREX1, ADAR1 and IFIH1 have been described [Livingston and Crow, 2016].

One of the variants in the current patient is likely to be degraded by non-sense mediated mRNA decay, while the p.(Phe165Ser) variant has been classified as a variant of unknown significance by ClinVar [Oleksy et al., 2022]. This variant locates within the deoxyribonucleoside triphosphate triphosphohydrolase catalytic core domain with dNTPase activity, single-stranded DNA/RNA binding activity, and exonuclease activity. Since this is likely the only expressed variant, it is plausible that it may inactivate these enzyme activities of the encoded protein. Thus, the p.(Phe165Ser) variant is presumed to cause the clinical syndrome by an autosomal recessive mechanism. In transgenic mice deficient for Samhd1, spontaneous transcription and translation of IFN- $\beta$ and its subsequent interferon-stimulated genes were found in the absence of any viral trigger [Martinez-Lopez et al., 2018]. Oleksy and colleagues suggest that the cystic brain lesions seen by MRI in their patient may be a result of the destructive effects of interferon on the developing brain in utero [Vanderver et al., 2015, La Piana et al., 2016; Oleksy et al., 2022].

The patient exhibited a neonatal onset of AGS, is severely handicapped, has spastic quadriplegia with an extrapyramidal component, and has acquired microcephaly [Oleksy et al., 2022]. A neonatal onset and a severe disease course, as well as severe neuroradiological features should raise the suspicion of mutations affecting TREX1, rather than SAMHD1 [Crow et al., 2015; La Piana et al., 2016; Livingston and Crow, 2016]. On the other hand, this patient did not show any of the features typically associated with $S A M H D 1$ variants, such as chilblains, intracerebral large artery disease, glaucoma or mouth ulcers [Dale et al., 2010; Ramesh et al., 2010; Crow et al., 2015]. Given the surprising findings in this patient, a clinical diagnosis of AGS, and especially an AGS subtype, is not straightforward as is evident from a table with shared and differentiating clinical features of AGS patients as a function of the underlying variant(s) [Oleksy et al., 2022]. This case re-emphasizes the need for thorough clinical investigation combined with genome-wide searches for genomic variants. Clearly, the clinical and the genome investigations cannot substitute for each other. This study, based on a single patient, contributes novel insights into the phenotypic ramifications of specific subtypes of AGS and improves our understanding of this disorder.

Martin Poot

\section{References}

Aicardi J, Goutières F. A progressive familial encephalopathy in infancy with calcifications of the basal ganglia and chronic cerebrospinal fluid lymphocytosis. Ann Neurol. 1984 Jan;15(1):49-54.

Crow YJ, Chase DS, Lowenstein Schmidt J, Szynkiewicz M, Forte GM, Gornall HL, et al. Characterization of human disease phenotypes associated with mutations in TREX1, RNASEH2A, RNASEH2B, RNASEH2C, SAMHD1, ADAR, and IFIH1. Am J Med Genet A. 2015 Feb;167A(2):296-312.

Crow YJ, Hayward BE, Parmar R, Robins P, Leitch A, Ali M, et al. Mutations in the gene encoding the $3^{\prime}-5^{\prime}$ DNA exonuclease TREX1 cause Aicardi-Goutières syndrome at the AGS1 locus. Nat Genet. 2006a Aug;38(8):917-20.
Crow YJ, Leitch A, Hayward BE, Garner A, Parmar R, Griffith E, et al. Mutations in genes encoding ribonuclease $\mathrm{H} 2$ subunits cause Aicardi-Goutières syndrome and mimic congenital viral brain infection. Nat Genet. 2006b Aug;38(8):910-6.

Dale RC, Gornall H, Singh-Grewal D, Alcausin M, Rice GI, Crow YJ. Familial Aicardi-Goutières syndrome due to SAMHD1 mutations is associated with chronic arthropathy and contractures. Am J Med Genet A. 2010 Apr;152A(4):938-42.

Fitzpatrick D, Holmes NE, Hui L. A systematic review of maternal TORCH serology as a screen for suspected fetal infection. Prenat Diagn. 2022 Jan;42(1):87-96.
La Piana R, Uggetti C, Roncarolo F, Vanderver A, Olivieri I, Tonduti D, et al. Neuroradiologic patterns and novel imaging findings in Aicardi-Goutières syndrome. Neurology. 2016 Jan;86(1):28-35.

Lebon P, Badoual J, Ponsot G, Goutières F, Hémeury-Cukier F, Aicardi J. Intrathecal synthesis of interferon-alpha in infants with progressive familial encephalopathy. J Neurol Sci. 1988 Apr;84(2-3):201-8.

Livingston JH, Crow YJ. Neurologic Phenotypes Associated with Mutations in TREX1, RNASEH2A, RNASEH2B, RNASEH2C, SAMHD1, ADAR1, and IFIH1: Aicardi-Goutières Syndrome and Beyond. Neuropediatrics. 2016 Dec;47(6):355-60. 
Oleksy B, Mierzewska H, Tryfon J, Wypchło M, Wasilewska K, Zalewska-Miszkurka Z, et al. Aicardi-Goutières Syndrome due to the SAMHD1 mutation presenting with deep white matter cysts. Mol Syndromol. 2022; doi:10.1159/000518941.

Ramesh V, Bernardi B, Stafa A, Garone C, Franzoni $\mathrm{E}$, Abinun $\mathrm{M}$, et al. Intracerebral large artery disease in Aicardi-Goutières syndrome implicates SAMHD1 in vascular homeostasis. Dev Med Child Neurol. 2010 Aug;52(8):72532.
Rice GI, Bond J, Asipu A, Brunette RL, Manfield IW, Carr IM, et al. Mutations involved in Aicardi-Goutières syndrome implicate SAM$\mathrm{HD} 1$ as regulator of the innate immune response. Nat Genet. 2009 Jul;41(7):829-32.

Rice GI, Del Toro Duany Y, Jenkinson EM, Forte GM, Anderson BH, Ariaudo G, et al. Gain-offunction mutations in IFIH1 cause a spectrum of human disease phenotypes associated with upregulated type I interferon signaling. Nat Genet. 2014 May;46(5):503-9.
Rice GI, Kasher PR, Forte GM, Mannion NM, Greenwood SM, Szynkiewicz M, et al. Mutations in ADAR1 cause Aicardi-Goutières syndrome associated with a type I interferon signature. Nat Genet. 2012 Nov;44(11):12438.

Vanderver A, Prust M, Kadom N, Demarest S, Crow YJ, Helman G, et al. Early-Onset Aicardi-Goutières Syndrome: Magnetic Resonance Imaging (MRI) Pattern Recognition. J Child Neurol. 2015 Sep;30(10):1343-8. 